\title{
Argumentation structures that integrate dialectical and non-dialectical reasoning
}

\author{
ANDREW STRANIERI, ${ }^{1}$ JOHN ZELEZNIKOW ${ }^{2}$ and \\ JOHN YEARW OOD \\ ${ }^{1}$ Donald Berman Laboratory for Information Technology and Law, Applied Computing Research Institute, La Trobe University, \\ Bundoora, Victoria, 3083, Australia; e-mail: stranier@.cs.latrobe.edu.au \\ ${ }^{2}$ Joseph Bell Centre for Forensic Statistics and Legal Reasoning, Faculty of Law, University of Edinburgh, Scotland, UK; e- \\ mail: john.zeleznikow@ed.ac.uk \\ ${ }^{3}$ School of Information Technology and Mathematical Sciences, University of Ballarat, Victoria, 3350, Australia; e-mail: \\ j.yearwood@ballarat.edu.au
}

\begin{abstract}
Argumentation concepts have been applied to numerous knowledge engineering endeavours in recent years. For example, a variety of logics have been developed to represent argumentation in the context of a dialectical situation such as a dialogue. In contrast to the dialectical approach, argumentation has also been used to structure knowledge. This can be seen as a non-dialectical approach. The Toulmin argument structure has often been used to structure knowledge non-dialectically yet most studies that apply the Toulmin structure do not use the original structure but vary one or more components. Variations to the Toulmin structure can be understood as different ways to integrate a dialectical perspective with a non-dialectical one. Drawing the dialectical/non-dialectical distinction enables the specification of a framework called the generic actual argument model that is expressly non-dialectical. The framework enables the development of knowledge-based systems that integrate a variety of inference procedures, combine information retrieval with reasoning and facilitate automated document drafting. Furthermore, the non-dialectical framework provides the foundation for simple dialectical models. Systems based on our approach have been developed in family law, refugee law, determining eligibility for government legal aid, copyright law and e-tourism.
\end{abstract}

\section{Introduction}

According to JB Freeman (1991), argumentation involves a family of concepts that can be broadly grouped into three categories: concepts related to the process of engaging in an argument, procedures or rules adopted to regulate the argument and argument as a product or artefact of an argument process. The first two categories, process and procedures, are intimately linked to a dialectical situation within a community of social agents.

JB Freeman (1991 at p. 20) defines a dialectical situation as "one that involves some opposition among participants to a discourse over some claim, that it involves interactive questioning for critically testing this claim and this process proceeds in a regimented, rule governed manner". A dialectical situation need not occur between two independent human agents in that monologues can be represented dialectically. For instance, a mathematician engaged in a solo demonstration that a proposition follows from axioms does not overtly engage in a discourse. Nevertheless the reasoning can be seen as a linguistic reconstruction of an imaginary discursive exchange within a community of mathematicians.

Argumentation as product involves viewing the linguistic reconstruction of what the argumentation process and procedure have generated. It involves laying out the premises, claims and layout of claims. 
For JB Freeman (1991), the distinction between the three views of argumentation - process, procedure and product - is largely illusory and unnecessarily confusing, particularly for his objective of identifying diagramming techniques for the clear articulation of arguments.

Argumentation concepts have been applied in recent years in a variety of knowledge engineering applications, typically without a clear delineation of argumentation as process, procedure or product. The central claim advanced in this article is that benefits inherent in the use of argumentation frameworks for information system knowledge engineering can be substantially enhanced if key features of the distinction between argumentation as process, procedure and product are maintained.

In this paper, the label dialectical argumentation is used to describe the modelling of discourse. This is contrasted with non-dialectical argumentation. Argumentation as dialectic (process and procedure) models situations that involve discourse within a community of agents. The agents need not be independent human agents engaged in group discussion but may even be a single software agent that has internal processes that involve dialectical exchange. In contrast, non-dialectic argumentation describes the use of argumentation to order, organise or structure knowledge without directly modelling a dialectical exchange.

Until recent decades, argumentation theories have been advanced for philosophical pursuits and not specifically to enhance knowledge engineering. As a consequence, the distinction between the dialectical and non-dialectical use of argumentation concepts is rarely prominent. For example, Aristotle presented three types of arguments: demonstrations, dialectical deductions and contentious deductions (Topics, Book 1, 100a 27-30). Although each of Aristotle's three types of argument can be seen as arising out of discursive exchanges, there is an implicit emphasis on the dialectical perspective for dialectical deductions because these arguments are made on the basis of premises that are debatable. They typically concern opinions that are adhered to with variable intensity by community members whereas demonstrations are assumed to have more of a ring of universal acceptance. Demonstrations are arguments whose claims are made from premises that are true and primary known, in more modern terminology, as analytic proofs. Contentious deductions are arguments that appear acceptable at first sight but, upon closer inspection, are not.

The analysis of argument advanced by Toulmin (1958) does not distinguish dialectical from nondialectical argumentation. By illustrating that logic could be seen as a kind of generalised jurisprudence rather than as a science, Toulmin (1958) advanced a structure of argument that captures the layout of arguments. Jurisprudence focuses attention on procedures by which legal claims are advanced and attacked and, in a similar way, Toulmin sought to identify procedures by which any claim, in general, is advanced. He identified a layout of arguments that was constant regardless of the content of the argument.

Toulmin (1958) concluded that most arguments, regardless of the domain, have a structure that consists of six basic invariants: claim, data, modality, rebuttal, warrant and backing. Every argument makes a claim based on some data. The argument in Figure 1 is drawn from reasoning regarding refugee status according to the 1951 United Nations Convention relating to the Status of Refugees (as amended by the 1967 United Nations Protocol relating to the Status of Refugees), and relevant High

\begin{tabular}{c} 
Reff has a real chance of \\
persucution \\
\cline { 2 - 3 } $\begin{array}{c}\text { Relocation within the country } \\
\text { of origin is unlikely to lead to } \\
\text { protection } \\
\text { BACKING }\end{array}$
\end{tabular}

Figure 1 Toulmin argument for well-founded fear 
Court of Australia rulings. The claim of the argument in Figure 1 is the statement that Reff has a wellfounded fear of persecution. This claim is made on the basis of two data items: that Reff has a real chance of persecution and that relocation within Reff's country of origin is not appropriate. A mechanism is required to act as a justification for why the claim follows from data. This justification is known as the warrant which is, in Figure 1, the statement that "the test for well-founded fear is real chance of persecution unless relocation affords protection". The backing provides authority for the warrant and in a legal argument is typically a reference to a statute or a precedent case. The rebuttal component specifies an exception or condition that obviates the claim. Reff may well have a real chance of persecution and relocation is unlikely; however, the claim that his fear is well founded does not hold if Reff's persecution is due to criminal activities.

The central theme of this paper is that a distinction between argument as process and procedure, called here dialectical, and argument as product called non-dialectical serves useful purposes for knowledge engineering in that it has motivated the development of a knowledge representation framework that clearly separates the two perspectives. A framework for knowledge engineering that supports the non-dialectical perspective expressively is described. The non-dialectical framework called the Generic Actual Argument Model (GAAM) directly facilitates the development of hybrid systems, intelligent document drafting, data mining and intelligent information retrieval. Furthermore, the non-dialectical framework provides a knowledge representation base that is the foundation for dialectical models.

The Generic Actual Argument Model (GAAM) is a variant of the layout of arguments advanced by Toulmin (1958). Arguments for non-dialectical purposes are represented at two levels of abstraction: the generic and the actual level. The generic level is sufficiently general so as to represent claims made by all members of a discursive community. All participants use the same generic arguments to construct, by instantiation, their own actual arguments. The generic arguments represent a detailed layout of arguments acceptable to all participants whereas the actual arguments capture a participant's position with respect to each argument. The actual arguments that one participant advances are more easily compared with those advanced by another, in a dialectical exercise, because in both cases the actual arguments have been derived from a generic template that all participants share.

The validity of the dialectical/non-dialectical distinction for knowledge engineering is demonstrated in the next section by noting that many applications of the Toulmin structure to knowledge modelling during the last decade have varied the structure in one way or another. However ad hoc the variations seem at first sight, they can be understood if seen as attempts to emphasise the dialectical as opposed to the non-dialectical perspective, to different extents.

In Section 3, the GAAM is presented. By specifically attempting to develop a non-dialectical model at a level that is generic to a discursive community, a variation of the Toulmin structure is derived that does not itself model dialectical exchanges. Rather, it enables dialectical exchanges to be readily modelled once communal knowledge is organised using the non-dialectical model. Applications developed with the use of the GAAM are discussed in Section 4 together with some insights regarding the dialectical model that is to be developed on the basis of the non-dialectical frame.

\section{Variations of Toulmin's structure}

Argumentation has been used in knowledge engineering in two distinct ways: with a focus on the use of argumentation to structure knowledge (i.e. non-dialectical emphasis) and with a focus on the use of argumentation to model discourse (i.e. dialectical emphasis). Dialectical approaches typically automate the construction of an argument and counter arguments normally with the use of a nonmonotonic logic where operators are defined to implement discursive primitives such as attack, rebut, or accept. Carbogim et al. (2000) present a comprehensive survey of defeasible argumentation.

Dialectical models have been advanced by Cohen (1985); Fox (1986); Vreeswijk (1993); Dung (1995); Prakken (1993a, 1993b); Prakken and Sartor (1996); Gordon (1995); Fox and Parsons (1998) 
and many others. In general these approaches include a concept of conflict between arguments and the notion that some arguments defeat others. Most applications that follow a dialectical approach represent knowledge as first-order predicate clauses, though they engage a non-monotonic logic to allow contradictory clauses. Mechanisms are typically required to identify implausible arguments and to evaluate the better argument of two or more plausible ones. For example, Fox and Parsons (1998) analyse and extend the non-standard logic LA of Krause et al. (1995). In that formalisation, an argument is a tuple with three components: (Sentence : Grounds : Sign). The sentence is the Toulmin claim, though this may be a simple claim or a rule. The sign is a number or symbol that indicates the confidence warranted in the claim. The grounds are the sentences involved in asserting the claim and can be seen as the reasoning steps used to ultimately reach the conclusion.

The preference for one argument over others has been modelled in a variety of ways. Prakken (1993a, 1993b) extends the framework proposed by Poole (1988) by using a concept of specificity. The claim that a penguin flies because it is a bird and all birds fly is less specific than the claim that a penguin does not fly. Preference relations between rules are elicited from experts and explicitly specified in the defeasible reasoning logic described by Antoniou (1997).

In applications of argumentation to model dialectical reasoning, argumentation is used specifically to model discourse and only indirectly used to structure knowledge. Concepts of conflict and of argument preferences map directly onto a discursive situation where participants are engaged in dispute. In contrast, many uses of argumentation for knowledge engineering application do not model discourse. This corresponds more closely to a non-dialectical perspective.

A non-dialectical representation facilitated the organisation of complex legal knowledge for information retrieval by Dick $(1987,1991)$. She illustrates how relevant cases for an information retrieval query can be retrieved despite sharing no surface features if the arguments used in case judgements are represented as Toulmin structures. Marshall (1989); Ball (1994) and Loui et al. (1997) have built hypertext-based computer implementations that draw on knowledge organised as Toulmin arguments. Hypertext links connect an argument's assertions with the warrants, backing and data of the same argument and also link the data of one argument with the assertion of other arguments. In this way, complex reasoning can be represented succinctly, enabling convenient search and retrieval of relevant information.

Clark (1991) represented the opinions of individual geologists as Toulmin structures so that his group decision support system could identify points of disagreement between experts. Matthijssen (1999) provides a further example of benefits that arise from the use of the original Toulmin structure. He represented user tasks as Toulmin arguments and associated a list of keywords to the structure. These keywords were used as information retrieval queries into a range of databases. Results indicate considerable advantages in precision and recall of documents as a result of this approach compared with approaches that require the user to invent queries.

Johnson et al. (1993) identified different types of expertise using this structure and Bench-Capon et al. (1991) used TAS to explain logic programming conclusions. Branting (1994) expands TAS warrants as a model of the legal concept of ratio decidendi. In the Split Up project, Zeleznikow and Stranieri (1995) and Stranieri et al. (1999) used TAS to represent family law knowledge in a manner that facilitated rule/neural hybrid development.

Toulmin proposed his views on argumentation informally and never claimed to have advanced a theory of argumentation. He does not rigorously define key terms such as warrant and backing. He loosely specifies how arguments relate to other arguments and provides no guidance as to how to evaluate the best argument or identify implausible ones. Nevertheless, the structure was found to be useful as a tool for organising knowledge.

According to JB Freeman (1991), the Toulmin layout does not explicitly model discourse. Operators to question, attack or qualify opposition assertions are not explicit. Nor is there a facility to represent an agent's beliefs as they differ from another agent's. Not surprisingly, many knowledge engineering applications of the Toulmin framework have not modelled discursive exchanges at all, but have applied the framework to structure knowledge. 
Despite the immediate appeal of TAS as a convenient frame for structuring knowledge, most researchers that use the Toulmin layout vary the original structure. Each variation can be seen to be an attempt to integrate some aspects of dialectical reasoning into a structure that, for knowledge engineering purposes, is largely non-dialectical. In the following section three variations are presented. These can be understood as attempts to integrate a dialectical approach into a non-dialectical one.

\subsection{Johnson's variation of the Toulmin layout}

Johnson et al. (1993) claim that any argument's backing can be classified into one of five distinct types of backing which they label type 1 to type 5. Each type of backing corresponds to a distinct type of expertise and also to a particular philosophical paradigm of reasoning, as follows.

- Type 1 arguments reflect axiomatic reasoning. Data and claim for these arguments are analytic truths. The supporting evidence derives from a system of axioms such as Peano's axioms of arithmetic. Examples of what Aristotle called demonstrations would be captured as type 1 arguments.

- Type 2 arguments assert a particular medical diagnosis on the basis of empirical judgements from a number of patients who have presented with similar symptoms in the past.

- Type 3 arguments are characterised by backings that reflect alternate representations of a problem. A medical diagnosis based on a model of the heart as a pump analyses symptoms to be consistent with that model. An alternate presentation that has the heart as a muscle provides other evidence.

- Type 4 arguments differ from type 3 arguments in that the alternate representations are conflicting. In this case the argument involves supporting evidence that is conflicting. An assertion is made by creating a composite representation from conflicting ones.

- Type 5 backings refer to paradigms that reflect a process of enquiry.

The type 1 and 2 backings that Johnson et al. (1993) identify are markedly different from types 3, 4 and 5. In the latter group, a claim is ultimately backed by recourse to alternate representations of a problem. The resolution of conflicting representations is akin to a dialectical process. A common solution is sought from the exchange that is stimulated from conflicting representations. In type 1 (axiomatic) or type 2 (empirical) arguments, the backing is made from one perspective. There are no alternate representations and no common solutions. This is an example of a non-dialectical perspective.

The Johnson et al. (1993) variation does not introduce or eliminate components of the original Toulmin layout. However, by discerning non-dialectical backings from dialectical ones, it imposes a typology of backing that can be seen as an attempt to extend the structure toward somewhat of a dialectical application. The approach is limited by the unclear nature of the Toulmin warrant.

Broadly speaking, Toulmin formulates the warrant as an inference procedure. It is a procedure for inferring a claim given data. For example, the statement that "most Italians are Catholic" can be used as an inference rule to infer the claim that Mario is (probably) a Catholic given data that he is an Italian. However, the statement that "most Italians are Catholic" can also be interpreted as a reason for the relevance of the data item "Mario is an Italian" in the argument.

The distinction between a warrant as an inference rule and a warrant as a reason for relevance can be seen in the refugee argument of Figure 1. The warrant statement that reflects that the High Court case of Chan introduced a "real chance of persecution" as the test for well-founded fear is readily seen as a reason for the relevance of the real chance data item. It is less obviously viewed as an inference rule that can be applied to infer the claim.

Below, issues related to what JB Freeman (1991) calls the problematic notion of warrant are discussed. However, it is important to note the Johnson typology applies to backings for warrants that are inference procedures but may not apply in the same way to warrants that are statements indicating a reason for the relevance of a data item. 


\subsection{The Freeman/Farley variation on Toulmin warrants}

Farley and K Freeman (1995) recognised the need to extend the warrant component in order to develop a model of dialectical reasoning more formal than that proposed by Toulmin. Their main objective was to develop a system that could model the burden of proof concept in legal reasoning. The concept of burden of proof is often used to refer to the onus a discourse participant has to supply evidence. So, as Prakken (2001) notes in modelling this form of burden of proof using a dialogue game model, a judge directs the pleadings phase of proceedings by requiring one litigant or another to supply evidence to support their claims. However, the form of burden of proof that was the focus of attention for Farley and K Freeman (1995) involves the extent to which evidence is required in order to draw a conclusion. This varies with the severity of the misdemeanour. Except as otherwise provided by the law, the burden of proof requires proof by a preponderance of the evidence. In a criminal case, the state must prove all elements of the crime to a beyond reasonable doubt level. In cases of tax fraud, the burden of proof in a tax case is generally on the taxpayer (Black, 1990).

K Freeman (1994) describes two types of warrants she called wtype1 and wtype2. The first warrant type, wtype1, classifies the relationship between assertion and data with category labels she calls explanatory or sign. Causal links are examples of explanatory warrants because they explain an assertion given data. Fire causes smoke. The consequent is explained by recourse to a cause-effect link. Other types of explanatory warrant include definitional relationships or property-attribute relationships. A sign relationship represents a correlational link between data and assertion.

The second warrant type, wtype2, represents the strength with which the assertion can be drawn from data. Examples of this type of warrant proposed by Freeman represent the strength with which the consequent can be drawn from the antecedent. Default type warrants represent default relationships such as "birds fly". Evidential warrants are less certain. Sufficient warrants are certain and typically stem from definitions.

Freeman explicitly represents reasoning methods in addition to the two types of warrant. The reasoning types reside outside the Toulmin structure but interact with warrants in order to produce credible outcomes. Her model incorporates four reasoning mechanisms: modus ponens, modus tolens, abduction and contra-positive abduction. For example, some reasoning mechanisms are stronger than others according to heuristics she devised. Modus ponens and modus tolens are assigned a strong link qualification if used with sufficient warrants, whereas the same reasoning types are assigned a "credible" qualification if used with evidential warrants.

Reasoning types interact with warrant types to control the generation of arguments according to reasoning heuristics. For example, modus ponens-abduction combinations are not permitted for two explanatory warrants unless both are evidential. K Freeman (1994) demonstrates a capacity her model has for dialectical reasoning. An assertion is initially argued for with the use of heuristics she defined. Then, an alternate argument is compared with the initial argument constructed and support for it is ascertained. The comparisons require the notion of level of proof which include beyond reasonable doubt, scintilla of evidence and preponderance of evidence.

Freeman's model is a sophisticated extension to the Toulmin structures that displays impressive dialectical reasoning results. She advances types of relationship between consequents and antecedents (wtpye1) and assigns the link a strength (wtype2). The discernment of two types of warrant is essential for her because her model of burden of proof relies on it. By specifying reasoning types and heuristics for their interaction with warrants, Farley and K Freeman (1995) can be seen to provide a way to extend the Toulmin structure so that it can be applied to model dialogue. The ambiguity in the original Toulmin warrant is dealt with by reserving one type of warrant for the inference rule and the other to indicate the strength of the rule. This adds a representation of uncertainty to some extent but, as we shall describe below, the strength of the data items and strength of claims are not represented. Furthermore, there is no attempt to incorporate information regarding the broader context of the argument. 
In contrast, the issue of context is paramount for Bench-Capon (1998), who is not intent on modelling the burden of proof in legal reasoning but on implementing a dialogue game that engages players in constructing arguments for and against assertions initially made by one party.

\subsection{Bench-Capon's variation}

Bench-Capon (1998) does not distinguish types of backing as Johnson et al. (1993) do, or types of warrant following Farley and K Freeman (1995). Instead, he introduces an additional component to the TAS. The presupposition component of a TAS represents assumptions made that are necessary for the argument but are not the object of dispute and so remain outside the core of the argument. A presupposition for the refugee argument illustrated in Figure 1 would indicate that the country in which the argument is raised is a signatory to the United Nations Convention. As Australia is a signatory to the Convention, the data items and warrant that relate to the UN Convention are entirely appropriate. If Australia were not a signatory then those data items may not be as appropriate. This is illustrated in Figure 2.

Making explicit presuppositions in the argument structure is important for the use Bench-Capon (1998) makes of TAS. A programme that plays the part of one or both players in a dialogue game is often exposed to utterances in discourse that represent presuppositions and are not central to the discussion at hand.

The presuppositions can become critical if parties to a game do not share them. Bench-Capon (1998) interprets the warrant as an inference procedure much as Toulmin originally did. The dialogue game does not directly add dialectical operators such as rebut, attack or accept into the structure but these are instead encoded into the control mechanism that represent the rules of the dialogue game. The inherent ambiguity in the Toulmin warrant is not addressed; however, the context of the argument is modelled by the addition of a presupposition component.

The three variations to the Toulmin structure presented here can be seen to be attempts at clarifying how the structure can be used within a dialogue. This objective motivated Johnson to add types of backing. Each new backing type derives from the use of arguments by a discursive community. Farley and K Freeman (1995) were more direct and developed specific reasoning heuristics so that an argument and counter-argument are constructed as they would be within a discursive community. Bench-Capon defined a dialogue game that regulated the dialogue between two players who each encode their utterances as Toulmin components.

In the next section a variation of the Toulmin structure is proposed that specifically aims to model the structure of arguments in a non-dialectical manner. This is done in a manner that is at a sufficiently

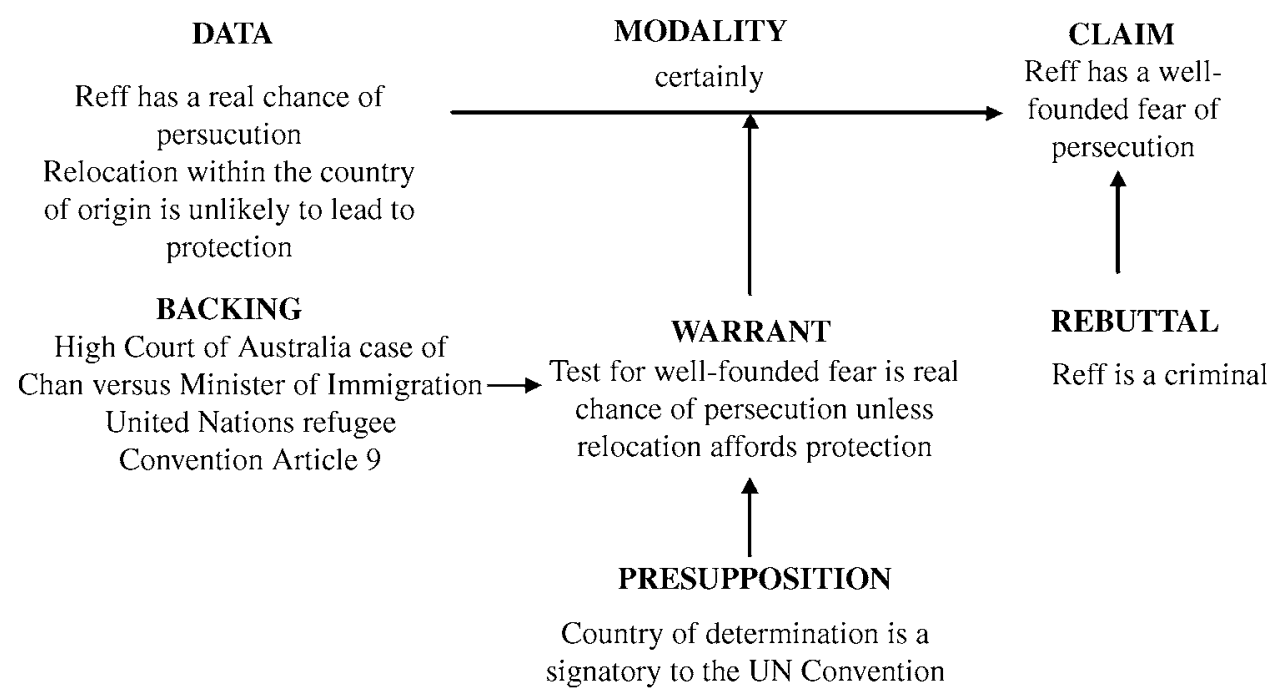

Figure 2 Toulmin argument structure with presupposition component 


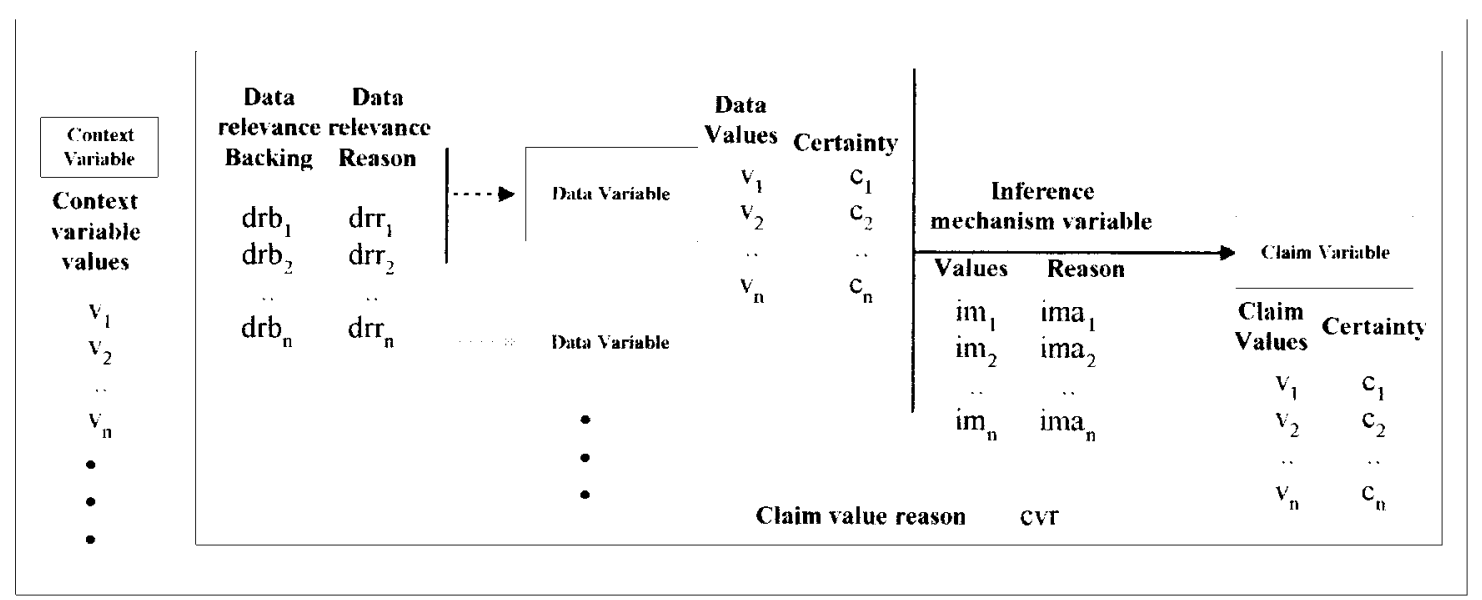

Figure 3 Non-dialectical argument template

high level of abstraction as to represent shared understanding between participants to a discourse which ultimately simplifies the specification of a dialectical model. However, even without extension into a dialectical model, the non-dialectical frame facilitates hybrid system development, document drafting and intelligent information retrieval.

\section{A generic non-dialectical model}

Figure 3 represents a template for knowledge representation that varies the Toulmin structure. The template differs from the Toulmin structure in that it includes

- a variable-value representation of claim and data items,

- a certainty variable associated with each variable-value rather than a modality or force associated with the entire argument,

- reasons for the relevance of the data items in place of the warrant,

- a list of inference procedures that can be used to infer a claim value from data values in place of the warrant,

- reasons for the appropriateness of each inference procedure,

- context variables,

- the absence of the rebuttal component present in the original formulation and

- the inclusion of a claim value reason component.

The argument template represents knowledge at a very high level of abstraction. There are two levels of instantiation made in applying the template to model arguments within a domain: the generic level and the actual level. A generic argument is an instantiation of the template where the following components are set:

- claim, data and context variables are specified but not assigned values,

- relevance reason statements and backing statements are specified,

- inference procedures are listed but a commitment to any one procedure is avoided,

- inference procedure reasons are specified for each procedure and

- claim and data variables are not assigned certainty values.

The generic argument is sufficiently general as to capture the variety of perspectives displayed by members of a discursive community.

Figure 4 illustrates the refugee argument above, as a generic argument. The claim variable has been labelled "well-founded fear" and acceptable values specified. There are three inference procedures known to be appropriate in this example; the first is a rule set that derives from heuristics an immigration expert uses, the second is a neural network trained from past cases and the third is a 


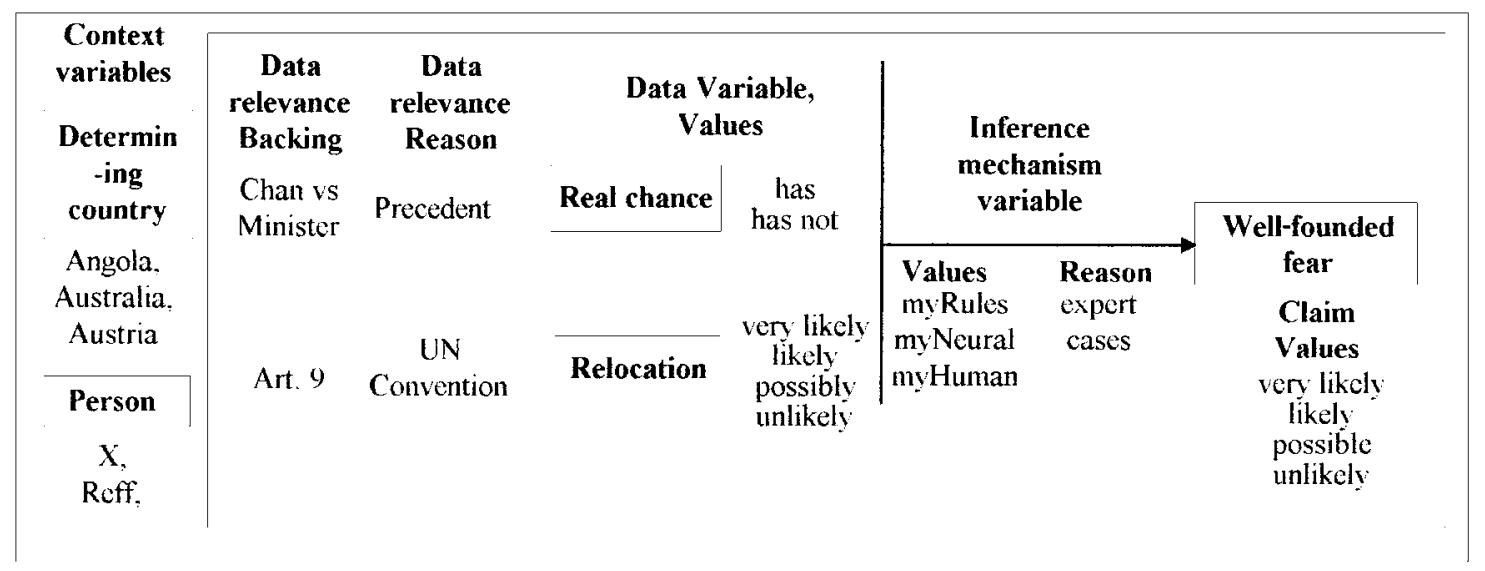

Figure 4 Generic argument for well-founded fear

human inference. This last inference indicates that a human is empowered with sufficient discretion to infer a claim value from data item values in any way he or she likes.

In the GAAM, the Toulmin warrant has been replaced with two components: an inference procedure and a reason for relevance. This relates to two different roles a warrant can play in an argument from a non-dialectical perspective. As described above, the warrant indicates a reason for the relevance of a data item and on the other hand the warrant can be interpreted as a rule which, when applied to the data items, leads to a claim inference.

An inference procedure is an algorithm or method used to infer a claim value from data item values. Under this interpretation, an inference procedure is a relation between data variable values and claim variable values. It is any procedure that will perform a mapping from data items to claim items. A mathematical function, an algorithm, a rule set, a neural network or procedures yet to be discovered are examples of inference procedures.

Many inference procedures can be implemented in software. Thus they can be automated in computer-based systems. However, this need not necessarily be the case for a knowledge engineering framework. Claims can sometimes be inferred from data items by human agents without the explicit specification of an inference procedure. This occurs frequently in discretionary fields of law where, as Christie (1990) notes, decision-makers weight and combine relevant factors in their own way without articulating precisely how claims were inferred. This situation is accommodated within the generic actual argument framework with the specification of an inference type labelled, simply, human.

The original Toulmin warrant can also be seen as a reason for relevance or an inference procedure. Past contributions to a marriage are relevant in Australian family law. Past contributions appears as a data item in a generic argument regarding property distribution following divorce because a statute dictates that contributions are relevant. The wealth level of a marriage in Australia is made relevant by past cases and not by statute. The hair colour of the wife is considered irrelevant because there is no statutory or precedent basis for its relevance. Further, domain experts can think of no reason that would make this feature relevant.

The concept of relevance is in itself difficult to define generally. Van Dijk (1989) describes the concept of relevance as it applies to a class of modal logics broadly called 'relevance logics' as a concept grounded firmly in the pragmatics, and not the semantics or syntax of language. Within a discursive community, the data items in a generic argument must be relevant to the claim to the satisfaction of members of the community.

A generic argument in the field of family law property division may include hair colour as a relevant data item for inferring property division if a reason for its relevance that is acceptable, even if not held, by many in the community, is advanced. Perhaps the utterance "blond women will remarry more readily" as a reason for the relevance of hair colour as a data item may not be held by all participants to a discourse but reflects a belief that is understood as plausible by many. 
The argumentation framework advanced here not only departs from the Toulmin formulation by distinguishing inference procedure from reason for relevance but it also represents context explicitly. Figure 4 illustrates two context variables; the determining country and the person about which the argument is being made. The respective values are a list of world nations for the determining country and Reff or the more universal X for the person.

Context variables represent something of the background knowledge that impacts on the generic argument. For example, the context variable determining country in Figure 4 represents a scope constraint on the argument. This indicates that an actual argument can be made based on the generic argument however the determining country sets a context for the argument. The context variable is an articulation of the presuppositions that underpin the generic argument.

The context variable can also represent the scope of variables used in the generic argument. For example, the person context variable will be assigned the value $\mathrm{X}$ for a discourse participant intent on making the more universal argument that relates to well-founded fear of anyone. The participant that restricts the argument to Reff does so be setting the context variable to Reff. In general, context is a difficult concept to define. In the framework defined here, context is defined as presupposition and variable scope. However, other definitions can also be accommodated as long as they can be captured as variable-value tuples.

There is no rebuttal component in the generic argument. The rebuttal is more clearly regarded to be a dialectical component and is therefore omitted from this essentially non-dialectical frame. For instance, discursive participants may create actual arguments as instances of the same generic argument in ways that are quite different from others. Participant A may assert a different claim value than B, yet have perfect agreement on all data item values because a different inference procedure was selected. Any discussion regarding this difference, including exchanges that make the point that the difference constitutes an attack, or exchanges that seek to defend A or B's assertion, or exchanges that seek to identify the stronger argument, involve dialectical exchange and are omitted from the nondialectical frame.

Figure 5 represents an actual argument. This is the second level instantiation of the argument template in Figure 3. An actual argument corresponds to a position held by a participant in a discourse. It is an instantiation of a generic argument. The context variable person in the generic argument is instantiated to "Reff", indicating that the claim only applies to him and not to others. The data item value in Figure 5 represents the situation that "Reff is likely to have a well-founded fear". The inference procedure for the actual argument is the ruleset called myRules. As a consequence of applying that ruleset, the claim value is instantiated to represent that Reff is likely to have well-founded fear.

The claim value reason for this actual argument provides a reason for the specific claim value inferred rather than other claim values. The claim value reason in Figure 5 expresses a reason for why well-founded fear is likely, given the data items and inference procedure selected. The claim value

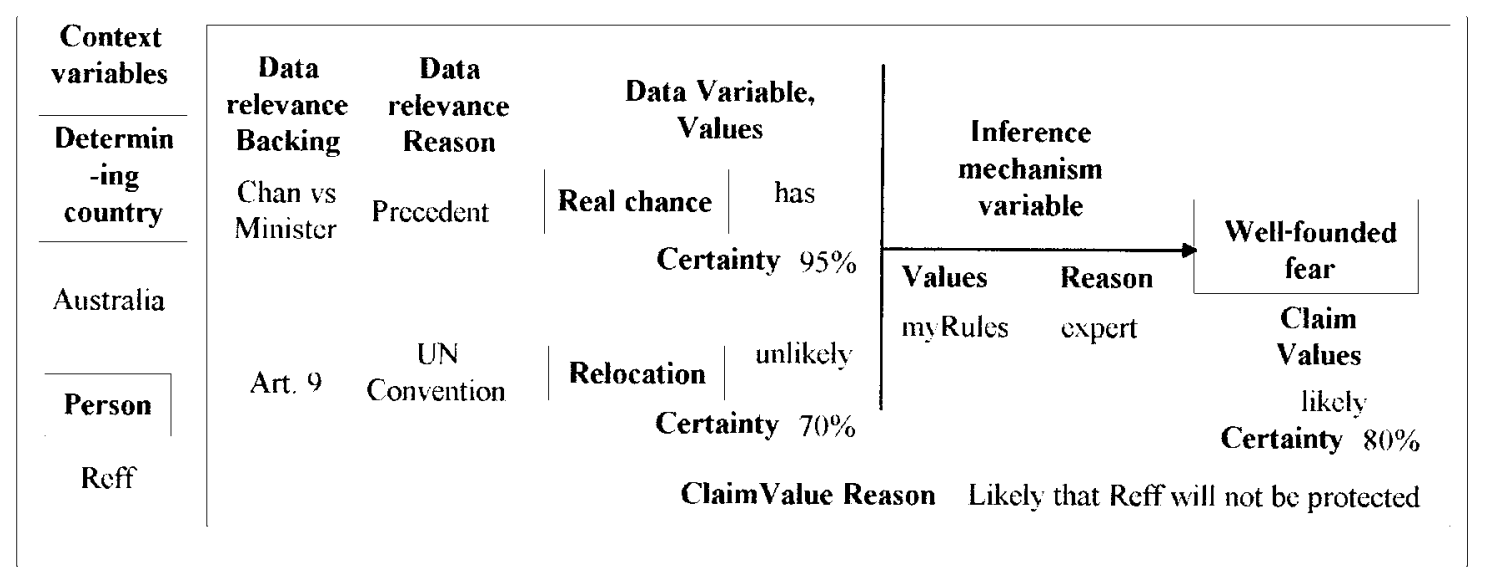

Figure 5 Actual argument for Reff has well-founded fear 
reason is not a reason for the inference rule. First of all the inference procedure need not be a rule. If it is a mathematical function or has mechanisms that are not visible, such as a neural network, then the articulation of a reason for the inference procedure is impossible. Conceptually, it is more correct to say it is a reason for a particular value that has arisen as a result of the application of an inference procedure.

Certainty values are assigned when a participant creates an actual argument. The certainty value represents the degree of certainty the participant has that the claim (or data) variable value selected is the true value. A certainty value may be set directly by the participant or calculated by the inference procedure, if the variable value is set by an inference procedure. The certainty value of $80 \%$ associated with the data item value, "likely" for the well-founded fear variable in Figure 5, is read as a high (80\%) degree of certainty that well-founded fear of persecution is likely. This is calculated by the inference procedure selected, myRules. However, if the inference procedure selected does not calculate certainty values (e.g. human inferences) then the participant must set a certainty value. The way in which the data item certainty values are combined is a feature of the mapping performed by the particular inference procedure selected and so is not made explicit in the GAAM.

Linguistic variable values such as very elderly, elderly, middle-aged, young and very young seem to represent certainty in themselves so as to make the specification of a certainty value redundant. However, the inclusion of a certainty value slot in the GAAM enables the specification of membership function values if fuzzy reasoning was selected as the inference procedure, conditional probability if a Bayesian inference was selected or certainty factors if Mycin-like rule inferences were used as the inference procedure.

Generic and actual argument structures correspond to a non-dialectical perspective. They do not directly model an exchange of views between discursive participants but rather describe assertions made from premises and the way in which multiple claims are organised. Claim variables are inferred using an inference procedure, which may not necessarily be automated, from data item values. The reasoning occurs within a context and the extent to which the data items correspond to true values, according to the proponent of the argument, is captured by certainty values.

The generic argument provides a level of abstraction that accommodates most points of view within a discursive community and anticipates the creation of actual arguments, by participants, as instantiations of a generic argument. However, it is conceivable that, given the open-textured nature of reasoning, that a participant will seek to advance an actual argument that is a departure from the generic argument. This is a manifestation of discretion and can be realised with the introduction of a new variable (data, claim or context) value, with the use of a new inference procedure or with a new claim value reason.

A non-dialectical argumentation model must model discretion and open texture. The concept of open texture was introduced by Waismann (1951) to assert that empirical concepts are necessarily indeterminate. A definition for open-textured terms cannot be advanced with absolute certainty unless terms are defined axiomatically, as they are, for example in mathematics. Gold may be defined as that substance which has spectral emission lines, $\mathrm{X}$ and is coloured deep yellow. However, because the possibility, that a substance with the same spectral emission as gold but without the colour of gold will appear in the future, cannot be ruled out the, concept for gold is open-textured.

The concept of open texture is significant in the legal domain because new uses for terms, and new situations, constantly arise in legal cases. Prakken (1993a) discerns three sources of open texture: reasoning which involves defeasible rules; vague terms; and classification ambiguities. Judicial discretion is conceptualised by Christie (1986) and Bayles (1990) as the flexibility decision-makers have in weighing relevant factors when exercising discretion, although articulating an assignment of weights is typically difficult. This view of discretion does not derive from defeasible rules, vague terms or classification ambiguities and so is regarded as a fourth type of situation that contributes to the opentextured nature of law.

The link between the GAAM and discretion is described in detail by Stranieri et al. (2000). Broadly, discretion manifests itself as the flexibility for a participant to construct an actual argument from a generic argument by 
- adding data item factors into the actual argument that are not in the generic tree,

- removing adata item factors from the actual argument that is in the generic tree,

- selecting a data, claim, or context variable value from those specified in the generic tree,

- selecting a data, claim, or context variable value that has not been specified in the generic tree,

- selecting an inference procedure from the list specified in the generic tree,

- selecting an inference procedure not specified in the generic tree,

- leaving data items, reasons for relevance, inference procedure, and reasons for the appropriateness of inference procedures implicit,

- introducing a claim value reason statement and

- selecting certainty values.

This framework including the generic/actual distinction, the clear separation of inference procedure from other components and the inclusion of reasons for relevance and context introduces a nondialectical structure that represents knowledge applicable to a discursive community but does not include elements that are clearly needed to model dialectical exchanges. In the next section, the way in which the specification of a comprehensive non-dialectical structure facilitates hybrid reasoning, document drafting and information retrieval is described before illustrating steps toward a dialectical model based on the GAAM non-dialectical frame.

\section{Applications of the generic/actual argument model}

The use of the GAAM for facilitating hybrid reasoning is illustrated with the knowledge-based system called Split Up, which predicts marital property distribution decisions following divorce made by judges of the Family Court of Australia. This research is reported by Stranieri et al. (1999).

\subsection{Split $U p$}

In the Split Up system, the relevant variables were structured as data and claim items, following the generic argument outlined above, into 35 interlocking arguments. The ultimate claim, representing the percentage split of assets a judge would be likely to award the husband and wife, was the root of an argument tree. Nodes in the tree, illustrated as Figure 6, are claim/data items. Variable values, inference procedures, reason for relevance and context are omitted from this diagram. The arguments interlock in that the claim of one argument is a data item for another higher up a tree such as the one depicted in Figure 6. For example, the variable contributions of the husband relative to the wife is a data item for the ultimate claim and also the claim for an argument that has four data items.

In the Split Up system, all claim variable values were inferred using automated inference procedures from the data item values. In 15 of the 35 arguments, claim values were inferred from data items with an inference procedure that involved the use of small rulesets that represent expert heuristics whereas neural networks, trained on data from past court cases, were used to infer claim values in the remaining 20 arguments.

The Split Up application illustrated that the generic/actual model captures knowledge in a way that leads to readily maintainable knowledge bases, a requirement that is particularly important in law. The tree of arguments underpinning Split Up was first elicited with the assistance of domain experts in 1994. Since then, property division in family law has changed in that domestic violence is now recognised as a relevant consideration in property proceedings following a divorce. The framework localises this change to a single argument that does not impact on any other argument. Furthermore, an examination of the process that led to the introduction of domestic violence illustrated that the generic argument framework can clarify judicial reasoning.

Alexander (2000) notes that during the last decade, numerous unsuccessful attempts were made to persuade judges to award a more generous property settlement to victims of domestic violence. This corresponds to the situation where an argument is advanced that departs from existing generic arguments by the introduction of a new data item. In recent years, a small number of Family Court judges began to accept the domestic violence argument. Many of the early cases were appealed and 


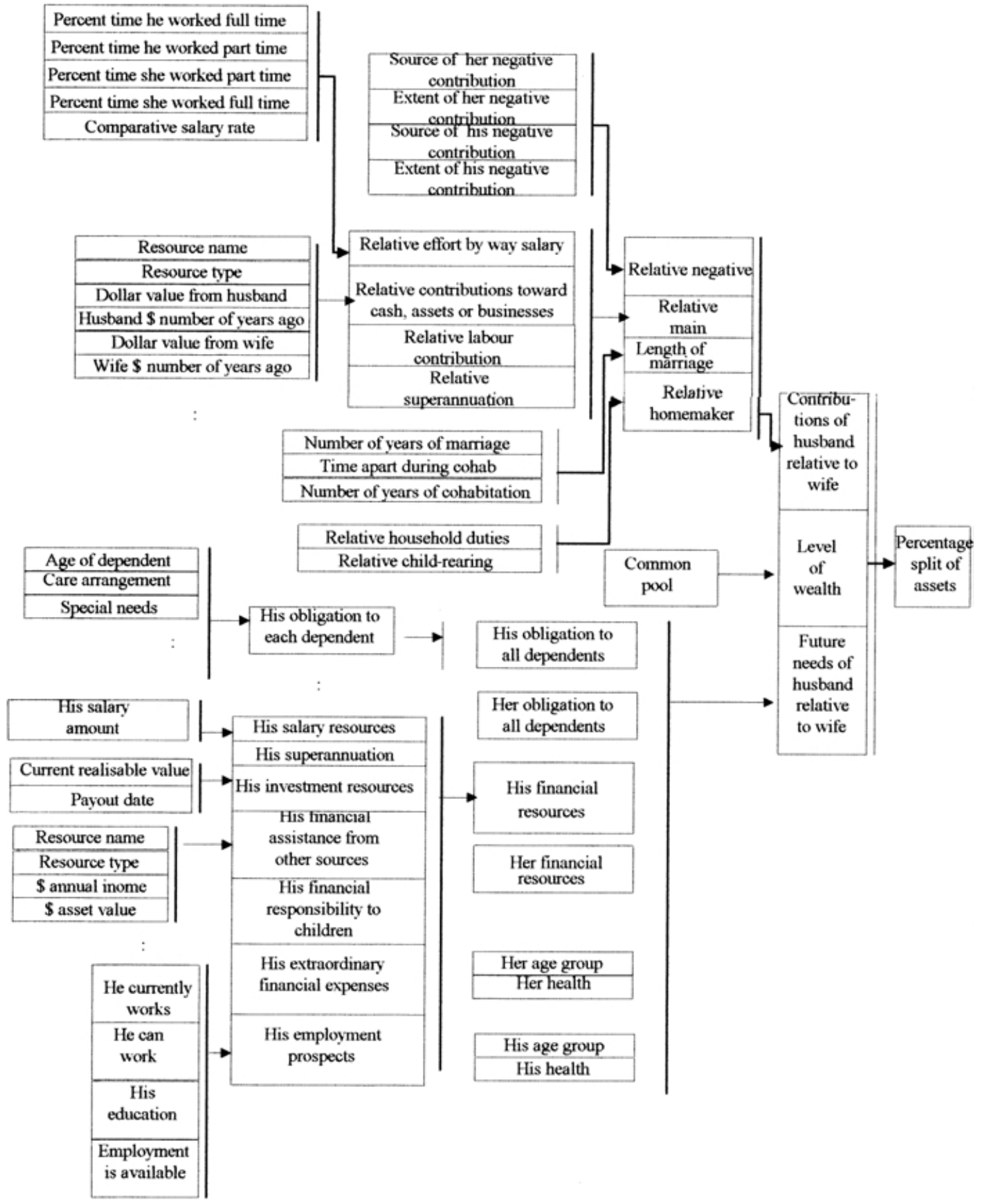

Figure 6 Split Up argument tree

precedents set by higher courts so that domestic violence is now undeniably a relevant consideration in property division. However, as Alexander (2000) notes, there is still some ambiguity in practice in that some judges have considered domestic violence a past contribution factor whereas others have recognised it as a factor that increases the victim's future needs. The ambiguity corresponds to a situation where a new factor has currently been inserted in two places in the argument tree. The discursive community, judges, lawyers and analysts of Australian family law, await the resolution of this apparent conflict.

The Split Up application also demonstrates that the generic/actual model provides a convenient frame for task decomposition that is particularly useful for data mining. Data mining is restricted to 
an exercise in discovering the inference procedure within each argument. Although the total number of relevant variables is large (103 in the Split Up system), most arguments have a small number of data items. Mining for an inference procedure involving a small number of variables is far more readily tractable than for a large set. Furthermore, a flat list of all variables requires huge numbers of cases and often includes missing values. For example, values associated with children are empty for childless marriages and appear in a flat list as null values. Null values severely hamper data-mining attempts. However, if the variables are organised into a generic tree, each argument has a small number of variables (data items). This means that relatively small numbers of cases can be used to discover inference procedures that are accurate.

Accurate inference procedures are particularly important in the Split Up systems because users are non-experts and need the system to prompt them for all relevant facts in order to infer all claims leading up to the culminating claim. This is in contrast to the Embrace system which is configured to make no automated inferences at all yet illustrates the document drafting and information retrieval benefits of the GAAM.

\subsection{Embrace}

Yearwood et al. (1999) report the application of the generic actual model to supporting reasoning regarding the assessment of refugee status in Australia. Refugee law is highly discretionary and extremely difficult to model. The main statute, the United Nations Convention, lists factors to be taken into account in reaching a determination on the refugee status of an applicant, but does not specify the weighting factors should have.

Ensuring that the decision-making is as consistent as possible in this complex and discretionary domain is critical for just outcomes. Yearwood et al. (1999) have modelled reasoning in this field using over 200 generic arguments derived from members of the body established to hear appeals from unsuccessful applicants, the Refugee Review Tribunal. Inference procedures have not been specified for any generic argument in order to ensure that the information system facilitates decision-making but does not directly infer outcomes which are left entirely to Tribunal members. Nevertheless, the argument structures have proven to be useful in modelling refugee decisions and in generating XML documents that are plausible first-draft determinations.

Refugee Review Tribunal determinations are documents that express the reasoning steps a member of the Tribunal followed in order to infer conclusions regarding the status of an applicant. Although it is reasonable to expect that a mapping between the reasoning steps used by judges and the structure of the judgement produced would clearly be apparent, Branting et al. (1999) note that such a mapping is by no means obvious. They make some progress beyond boilerplate templates with the sophisticated use of discourse analysis using speech act and rhetorical structure theory.

Yearwood and Stranieri (1999) have identified a simple heuristic for traversing a tree of actual arguments that leads to a plausible document structure. This is achieved without the use of discourse analysis methods largely because the generic/actual framework is a succinct, yet expressive, frame for capturing reasoning.

The document generation facility has been implemented as a module of an argument shell, called ArgumentDeveloper. Yearwood and Stranieri (2000) describe the program that has been written to facilitate the development of knowledge-based systems that use the generic actual model. This module traverses the actual argument tree for a user in the order specified by the algorithm and generates an XML document with an appropriate document type definition file. When this is paired with a stylesheet customised for refugee law, a determination is automatically generated that expresses the flow of reasoning in a manner that is quite plausible despite using no discourse-analysis techniques.

In addition to the need to generate draft determinations rapidly, the Embrace project provided the vehicle for demonstrating that the generic actual model improves information retrieval. This is implemented with the development of the information retrieval module into the ArgumentDeveloper shell. This module automatically generates a search engine query by assembling all terms used in an argument with a list of keywords associated with the argument. Matthijssen (1999) demonstrated 
improved precision and recall figures using keyword lists attached to the original Toulmin structure. The information retrieval query takes all variable names and values in addition to a list of terms associated with each generic argument in order to generate a query. Empirical trials are in progress to demonstrate the extent of precision recall improvements.

The system, called GetAid, operates in the field of legal aid eligibility where rapid prototyping of a web-based application is more important.

\subsection{GetAid}

The generic actual framework has been applied to acquire knowledge regarding decisions made by officers of Victoria Legal Aid, a government-funded provider of legal services for disadvantaged clients, in assessing whether an applicant should receive legal aid. Applicants for legal aid must pass a merits test that involves a prediction about the likely outcome of the case in court. This assessment involves considerable discretion and is performed by grants officers who have extensive experience in the practices of Victorian courts.

A web-based knowledge-based system called GetAid was rapidly developed using the WebShell, reported by Stranieri and Zeleznikow (2001a). Knowledge was modelled using two distinct techniques: decision trees for procedural type tasks and generic argument trees for tasks that are more complex, ambiguous or uncertain.

The GetAid development demonstrated that the generic actual argument model is a useful representation for rapid knowledge acquisition. In order to construct a generic argument tree, the expert is initially prompted to articulate factors (data item variables) that may be relevant in determining the ultimate claim without any concern about how the factors may combine to actually infer a claim value. For every factor (data item variable) articulated, a reason for the item's relevance must be able to be articulated. The possible values for each data item are then identified. The next step in the knowledge acquisition exercise involves viewing each data item as a claim and eliciting the data items that are used to infer its value.

Once the tree is developed as far back as the expert regards appropriate for the task at hand, attention can then be focused on identifying one or more inference procedures that may be used to infer a claim value from data item values. This proved difficult for the GetAid experts to articulate as rules because the way in which the factors combine is rarely made explicit but forms part of the expertise gathered over many cases. Although it is feasible to attempt to derive heuristics, the approach we used was to present a panel of experts with an exhaustive list of all combinations of data items as hypothetical cases and prompt for a likely decision. The decisions from a panel of experts were merged to form a dataset of records that were used to train a neural network for each generic argument.

The construction of the systems - GetAid, Split Up and Embrace - illustrate the benefits in the use of the non-dialectical framework. These include hybrid reasoning, task decomposition, information retrieval, document generation and knowledge acquisition. These benefits can be seen to derive from the effectiveness of the generic actual model to structure reasoning. In the e-tourism application, first steps have been made towards the development of a dialectical model that is based on the nondialectical model.

\subsection{E-tourism}

In the e-tourism system developed by Avery et al. (2001) knowledge is represented using the generic actual argument model. However, the aim of the system is to model the dialectical exchange between a tourist, tour operators and tourist advisors. The human tourist invokes an instance of a tourist software agent on commencing a consultation session. The tour advisor has no human counterpart. The dialogue between the tourist and advisor agents is aimed at realising the community goal of recommending tours the tourist will enjoy. The tour operator invokes an operator agent in order to inform the advisor of tours it operates. A key feature of the approach presented here is that all agents share the same generic argument tree but can instantiate their own actual arguments. In this way, each 
agent's beliefs are represented by actual arguments, but because these are instances drawn from a common generic argument tree, negotiation can be simplified.

Jennings et al. (2000) note that negotiation underpins any attempt at coordinating multiple agents (human or software). For instance, the architecture for the e-tourism application is based on an agentoriented approach where each software agent represents world knowledge as arguments and interacts with other agents according to dialogue rules. An agent-based framework that places emphasis on negotiation must include three main components: a negotiation protocol, a negotiation object and an agent decision-making model.

The generic argument constrains negotiation protocols in a convenient manner for agent-oriented architectures. The actual arguments of multiple agents can be readily compared and contrasted because each actual argument is an instantiation of the same generic argument. Operators that appear in dialectical argumentation such as attack and accept are readily implemented. An argument, A, attacks another argument, B, if A has a different claim value than B for the same claim variable. The source of the attack can be readily isolated. It may be due to different data item values, certainties or inference procedures. An argument, C, accepts another argument, D, if it has the same claim variable and value. Identical acceptance is operationalised as the same claim, data and inference procedures whereas similar acceptance occurs if the claims are the same but data or inferences are not. Research is currently in progress to develop the dialectical model based on the generic/actual split.

Stranieri and Zeleznikow (2001b) propose an agent-based knowledge-based approach to help regulate copyright. Five knowledge-based systems are described that are sufficiently flexible to protect authors' rights without denying the public access to works for fair-use purposes. The owner of a work and users who wish to copy a portion of the work are participants in the discursive community and share the same generic arguments. In order to copy the work users construct their own actual arguments. The agent representing the owner determines whether to release the work or not by constructing its own actual argument. The generic-actual framework simplifies the negotiation protocol and assists in the deployment of an agent-oriented approach.

\section{Conclusion}

Argumentation has been applied to knowledge engineering in recent years in two ways: with an emphasis on the dialectical nature of argumentation and with an emphasis on the structure of reasoning from a non-dialectical perspective. From the dialectical perspective, the way in which two or more participants in a discourse propose arguments that attack, rebut, defeat, subsume or accept others is paramount. From a non-dialectical perspective, the way in which claims are laid out and inferred from premises is the object of attention. The argument structure proposed by Toulmin (1958) does not clearly distinguish a dialectical perspective from one that is non-dialectical. Many applications of the Toulmin layout of arguments for knowledge engineering purposes vary the structure.

The variations made by K Freeman (1994), Bench-Capon (1998) and Johnson et al. (1993) can be understood as the result of different emphases on the dialectical or non-dialectical perspective, though in many cases the distinction is still blurred. A variation to the Toulmin structure, called the generic actual argument model, is advanced where the distinction between dialectical and non-dialectical argumentation concepts is clearly defined. The GAAM is a non-dialectical model that facilitates hybrid reasoning, information retrieval, document drafting, knowledge acquisition and data mining. The nondialectical GAAM has been applied to the construction of systems in refugee law, family law, eligibility for legal aid and copyright law. A dialectical model that is based on the GAAM is under investigation, though early results with the automated provision of e-tourism advice using an agent architecture indicate that a dialogue model is more readily realised simplified if built on the nondialectical base.

\section{References}

Alexander, R, 2000, "Reflections on gender in family law decision making in Australia" Ph.D thesis, Monash University, Australia. 
Antoniou, G 1997, Nonmonotonic Reasoning with Incomplete and Changing Information MIT Press.

Avery, J, Yearwood, J and Stranieri, A, 2001, "An argumentation based multi-agent system for etourism dialogue" First International Workshop on Hybrid Intelligent Systems (HIS'01) forthcoming.

Ball, WJ, 1994, "Using Virgil to analyse public policy arguments: a system based on Toulmin's informal logic" Social Science Computer Review 12(1) 26-37.

Bayles, MD, 1990, Procedural Justice. Allocating to Individuals Kluwer.

Bench-Capon, TJM, 1998, "Specification and implementation of Toulmin dialogue game" in JC Hage, T BenchCapon, A Koers, C de Vey Mestdagh and C Grutters (eds) Jurix 1998 Foundation for Legal Knowledge Based Systems Gerard Noodt Institut.

Bench-Capon TJM, Lowes, D and McEnery, AM, 1991, "Argument-based explanation of logic programs" Knowledge Based Systems 4(3) 177-183.

Black, HC, 1990, Black's Law Dictionary West Publishing Company.

Branting, KL, 1994, “A computational model of ratio decidendi" Artificial Intelligence and Law: an International Journal 2 1-31.

Branting, K, Callaway, C, Mott, B and Lester, J, 1999, "Integrating discourse and domain knowledge for document drafting" Proceedings of Seventh International Conference on Artificial Intelligence and Law 214-220.

Carbogim, D, Robertson, D and Lee, J, 2000, "Argument-based applications to knowledge engineering" Knowledge Engineering Review 15(2) 119-149.

Clark, P, 1991, "A model of argumentation and its application in a cooperative expert system" Ph.D. thesis, Turing Institute, Department of Computer Science, University of Strathclyde.

Cohen, P, 1985, Heuristic Reasoning about Uncertainty: An Artificial Intelligence Approach Pitman.

Christie, GC, 1986, "An essay on discretion” Duke Law Journal 747-78.

Dick, JP, 1987, "Conceptual retrieval and case law" Proceedings of the First International Conference on Artificial Intelligence and Law 106-115.

Dick, JP, 1991, “A conceptual, case-relation representation of text for intelligent retrieval” Ph.D. thesis, University of Toronto.

Dung, Phan Minh, 1995, "On the acceptability of arguments and its fundamental role in non-monotonic reasoning, logic programming and n-person games" Artificial Intelligence 77(2) 321-57.

Farley, AM and Freeman, K, 1995, "Burden of proof in legal argumentation" Proceedings of Fifth International Conference on Artificial Intelligence and Law 156-164.

Fox, J, 1986, "Knowledge, decision making and uncertainty" in WA Gale (ed.) Artificial Intelligence and Statistics Addison-Wesley.

Fox, J and Parsons, S, 1998, "Arguing about beliefs and actions" in A Hunter and S Parsons (eds) Applications of Uncertainty Formalisms Springer.

Freeman, JB, 1991, Dialectics and the Macrostructure of Arguments Floris Publications.

Freeman, K 1994, “Toward formalizing dialectical argumentation” Ph.D. thesis, Department of Computer Science and Information Science, University of Oregon.

Gordon, TF, 1995, "The pleadings game: an exercise in computational dialectics" Artificial Intelligence and Law 2(4) 239-92.

Jennings, N, Parsons, S, Sierra, C and Faratin, P, 2000, "Automated Negotiation" Proceedings of the fifth International Conference on The Practical Application of Intelligent Agents and Multi-Agent technology 23-30.

Johnson, PE, Zualkernan, IA and Tukey, D, 1993, "Types of expertise: an invariant of problem solving" International Journal of Man-Machine Studies 39641.

Krause, P, Ambler, S, Elvang-Gøransson, M and Fox, J, 1995, "A logic of argumentation for reasoning under uncertainty" Computational Intelligence 11(1) 113-131.

Loui, R, Norman, J, Altepeter, J, Pinkard, D, Craven, D, Lindsay, J and Foltz, M, 1997, "Progess on Room 5: a testbed for public interactive semi-formal legal argumentation" Proceedings of the Sixth International Conference on Artificial Intelligence and Law 207-214.

Marshall, CC, 1989, "Representing the structure of legal argument" Proceedings of Second International Conference on Artificial Intelligence and Law 121-127.

Matthijssen, LJ, 1999, Interfacing between lawyers and computers. An architecture for knowledge based interfaces to legal databases Kluwer Law International.

Poole, DL, 1988, “A logical framework for default reasoning” Artificial Intelligence 36 27-47.

Prakken, H, 1993a, "Logical tools for modelling legal argument" Ph.D. thesis, Vrije University.

Prakken, H, 1993b, "A logical framework for modelling legal argument" Proceedings of the Fourth International Conference on Artificial Intelligence and Law 1-9.

Prakken, H, 2001, "Modelling reasoning about evidence in legal procedure" Proceedings of the Eight International Conference on Artificial Intelligence and Law 119-128. 
Prakken, H and Sartor, G, 1996, "A dialectical model of assessing conflicting arguments in legal reasoning" Artificial Intelligence and Law 4 331-368.

Stranieri, A, Zeleznikow, J, Gawler, M and Lewis, B, 1999, "A hybrid rule - neural approach for the automation of legal reasoning in the discretionary domain of family law in Australia" Artificial Intelligence and Law 7(2-3) 153-183.

Stranieri, A and Zeleznikow, J, 2001a, "WebShell: the development of web based expert system shells" SGES British Expert Systems Conference (ES'01) 69-76.

Stranieri, A and Zeleznikow, J, 2001b, "Copyright regulation with argumentation agents" Journal of Information and Communication Law 10(1) 109-123.

Stranieri, A, Yearwood, J and Meikl, T, 2000, "The dependency of discretion and consistency on knowledge representation" International Review of Law, Computers and Technology 14(3) 325-340.

Toulmin, S 1958, The Uses of Argument Cambridge University Press.

Van Dijk, TA, 1989, "Relevance in logic and grammar" in J Norman and R Sylvan (eds) Directions in Relevant Logic. Kluer Academic Publishers, MA, USA.

Vreeswijk, G, 1993, "Defeasible dialectics: a controversy-oriented approach towards defeasible argumentation" Journal of Logic and Computation 3(3) 3-27.

Waismann, F, 1951, "Verifiability" in A Flew (ed.) Logic and Language Blackwell.

Yearwood, J, Stranieri, A and Anjaria, C, 1999, "The use of argumentation to assist in the generation of legal documents" ADCS'99 Fourth Australasian Document Computing Symposium 69-76.

Yearwood, J and Stranieri, A, 1999, "The integration of retrieval, reasoning and drafting for refugee law: a third generation legal knowledge based system" Seventh International Conference on Artificial Intelligence and Law (ICAIL'99) 117-137.

Yearwood, J and Stranieri, A, 2000, "An argumentation shell for knowledge based systems" Proceedings of IASTED International conference on Law and Technology 105-111.

Zeleznikow, J and Stranieri, A, 1995, "The Split Up system: integrating neural networks and rule based reasoning in the legal domain" Proceedings of the Fifth International Conference on Artificial Intelligence and Law (ICAIL'95) 185-194. 